\title{
SHARPENED FORMS OF A VON NEUMANN INEQUALITY FOR $\rho$-CONTRACTIONS
}

\author{
GILLES CASSIER and NICOLAE SUCIU
}

\begin{abstract}
The purpose of this paper is to give some sharpened forms of the von Neumann inequality for strict $\rho$-contractions which were obtained in [6], [7]. Also, some sharpened forms of the Schwarz inequality for strict $\rho$-contractions will be given, and as applications, corresponding inequalities for strict contractions and for uniformly stable operators will be derived. In particular, we recover the results of K. Fan [12], [13] covering the strict contractions, and in the scalar context we find an improved form of the interior Schwarz inequality quoted by R. Osserman [19].
\end{abstract}

\section{Introduction and preliminaries}

Let $H$ be a complex Hilbert space and $B(H)$ be the $C^{*}$-algebra of all bounded linear operators on $H$. For any scalar $\rho>0$, we denote by $C_{\rho}(H)$ the set of all operators $T \in B(H)$ which admit a unitary $\rho$-dilation in the sense of Nagy-Foias [21], [22]. This means that there exists a Hilbert space $\mathscr{H} \supseteq H$ and a unitary operator $U$ acting on $\mathscr{H}$ such that

$$
T^{n} h=\rho P_{H} U^{n} h \quad(h \in H, n \geq 1),
$$

where $P_{H}$ is the orthogonal projection of $\mathscr{H}$ onto $H$. We know that $C_{1}(H)$ consists of all contractions on $H$ [20], that is the operators $T$ on $H$ with $\|T\| \leq 1$, and that $T \in C_{2}(H)$ if and only if the numerical range of $T$ is contained in the closed unit disc [2].

According to J. Holbrook [15] and J. Williams [23] we define the $\rho$-numerical radius of an operator $T \in B(H)$ by the formula

$$
w_{\rho}(T)=\inf \left\{\mu: \mu>0, \frac{1}{\mu} T \in C_{\rho}(H)\right\} .
$$

Clearly, an operator $T$ belongs to $C_{\rho}(H)$ if and only if $w_{\rho}(T) \leq 1$. Hence the operators in $C_{\rho}(H)$ are contractions with respect to the $\rho$-numerical radius, and according to this fact, any operator $T \in C_{\rho}(H)$ will be called a $\rho$-contraction on $H$ (as in [4], [6]-[8], [9], [16], for instance). 
Let us remark that $w_{1}(T)=\|T\|$ and $w_{2}(T)=w(T)$ this being the numerical radius of $T$. Hence $C_{2}(H)$ consists of all operators with numerical radius less or equal to one ([2], [3], [21]). Also, since the classes $C_{\rho}(H)$ increase with $\rho$ ([3], [5], [21], [22]), one has $w_{\rho_{1}}(T) \geq w_{\rho_{2}}(T)$ if $\rho_{1} \leq \rho_{2}$ for $T \in B(H)$, and

$$
\lim _{\rho \rightarrow+\infty} w_{\rho}(T)=r(T)
$$

where $r(T)$ is the spectral radius of $T$.

Recall that if $T$ is an operator with $r(T)<1$ and if $f$ is an analytic function on the open unit disc $\mathrm{D}$ of the complex plane, then the operator $f(T)$ on $H$ is well-defined by the Riesz-Dunford integral [10]. Clearly, if $w_{\rho}(T)<1$ that is, $T$ is a strict $\rho$-contraction (see [6], [7]), then $r(T)<1$ and therefore $f(T)$ is well-defined for $f$ as above. Also, if $r(T)<1$ then $w_{\rho}(T)<1$ for $\rho>1$ large enough. Thus, many facts on the uniformly stable operators can be obtained from the strict $\rho$-contractions [6], [8].

It is well-known that an operator $T$ with $r(T) \leq 1$ belongs to $C_{\rho}(H)$ if and only if the following von Neumann equality holds

$$
\|f(T)\| \leq \sup _{|z| \leq 1}|\rho f(z)+(1-\rho) f(0)|
$$

for any analytic function $f$ on an open neighborhood of $\overline{\mathrm{D}}$. In fact, the inequality (1.1) for $T \in C_{\rho}(H)$ was essentially given by Sz.-Nagy-Foias [21], [22] and the converse assertion that the inequality (1.1) ensures $T \in C_{\rho}(H)$ was proved by D. Gaspar [14]. Clearly, the inequality (1.1) holds also for any continuous function $f$ on $\overline{\mathrm{D}}$ which is analytic on $\mathrm{D}$ (notation $f \in A(\mathrm{D})$ ) if $T \in C_{\rho}(H)$, where $f(T)$ is defined by setting $f(T)=\lim _{r \rightarrow 1^{-}} f(r T)$ in $B(H)((1.1)$ clearly implies the convergence of $f(r T)$ with respect to the norm of $B(H)$ )

An equivalent inequality to (1.1) can be obtained, namely

$$
w_{\rho}(f(T)) \leq\|f\|_{\infty}:=\sup _{|z| \leq 1}|f(z)|
$$

for $T \in C_{\rho}(H)$ and $f$ as in (1.1) with $f(0)=0$. Indeed, if (1.2) holds for $T \in B(H)$ with $r(T) \leq 1$ and for any function $f$ as above, then for $f(z)=z$, (1.2) implies $w_{\rho}(T) \leq 1$ that is, $T \in C_{\rho}(H)$ and consequently (1.1) holds for such a $T$. The converse implication is a consequence of the following version for $\rho$-contractions of the classical von Neumann theorem [18] for contractions, which was proved in [6].

THEOREM 1.1 (Mapping theorem). Let $f \in A(\mathrm{D})$ be a non-constant func- 
tion with $f(\mathrm{D}) \subseteq \mathrm{D}$. Then, for $T \in C_{\rho}(H)$ we have $f(T) \in C_{\rho_{f}}(H)$ where

$$
\rho_{f}= \begin{cases}1+(\rho-1) \frac{1-|f(0)|}{1+|f(0)|} & \text { if } \rho \leq 1 \\ 1+(\rho-1) \frac{1+|f(0)|}{1-|f(0)|} & \text { if } \rho \geq 1 .\end{cases}
$$

Moreover, if $\rho>0$ is the smallest scalar such that $T \in C_{\rho}(H)$ then there exists a function $f$ as above such that $\rho_{f}$ is the smallest positive scalar for which $f(T) \in C_{\rho_{f}}(H)$.

It is clear from (1.3) that $\rho=1$ implies $\rho_{f}=1$ for any $f$. Also, when $\rho \neq 1$ we have $\rho_{f}=\rho$ if and only if $f(0)=0$. Thus the inequality (1.2), and hence (1.1), can be derived from Theorem 1.1. In general one has $\rho \leq \rho_{f}$, and $\rho_{f} \leq 1$ if $\rho \leq 1$.

Using the fact that for any $\rho>0$ the mapping $S \rightarrow w_{\rho}(S)$ is norm continuous for $S \in B(H)$ (see [3]), we can derive (as in Corollary 8 [6]) the following useful result.

THEOREM 1.2. Let $f \in A(\mathrm{D})$ be a non-constant function with $f(\mathrm{D}) \subseteq \mathrm{D}$. Then, the inequality

$$
w_{\rho_{f}}(T)<1
$$

holds for every operator $T$ with $w_{\rho}(T)<1$, where $\rho_{f}$ is as in (1.3).

In the case $\rho=1$, from this theorem we deduce the well-known result of K. Fan [11] which is concerned with strict contractions. In [12], [13] K. Fan obtained some sharpened forms of von Neumann's inequality, as well as of Schwarz's inequality and of Schwarz-Pick inequality, for strict contractions.

In the present paper we sharpen the versions of Schwarz's inequality and of Schwarz-Pick's inequality given in [6], [7] for $\rho$-contractions. When $\rho=1$ we recover the results of K. Fan [12], [13], and in addition, we complete some results of $\mathrm{K}$. Fan in this case. We find also corresponding sharpened inequalities relative to the spectral radius, as consequences. Among others, we give an analogue of interior Schwarz inequality for $\rho$-contractions, which provides in the scalar case an improved form of the corresponding inequality proved by R. Osserman [19].

To obtain these inequalities, the following inequality proved in [6] (Theorem 5.8 and Remark 5.9) will be used more than once in our proofs. Namely, if $f$ is a non-constant analytic self map of $\mathrm{D}, \rho>0$ and $0<r<1$, then we 
have

$$
w_{\rho^{\prime}}(f(T)) \leq \sup _{|z|=r}|f(z)|:=M_{f}(r)
$$

for every operator $T$ on $H$ with $w_{\rho}(T) \leq r, \rho^{\prime}$ being as in (1.3) with $z \rightarrow$ $f(r z) / M_{f}(r)$ instead of $f$, that is $\rho^{\prime}=\rho_{f}(r)$ where

$$
\rho_{f}(r)= \begin{cases}1+(\rho-1) \frac{M_{f}(r)-|f(0)|}{M_{f}(r)+\mid f(0)} & \text { if } \rho \leq 1 \\ 1+(\rho-1) \frac{M_{f}(r)+|f(0)|}{M_{f}(r)-|f(0)|} & \text { if } \rho \geq 1 .\end{cases}
$$

Clearly, $\rho_{f}$ is related to the value $f(0)$ (by (1.3)), but we also apply (1.5) relatively to any point $a \in \mathrm{D}$ with $\rho(a)$ instead of $\rho>0$ and $\rho_{f}(a, r)$ instead of $\rho_{f}$, where

$$
\begin{aligned}
& \rho(a)=\rho_{\varphi_{a}}= \begin{cases}1+(\rho-1) \frac{1-|a|}{1+|a|} & \text { if } \rho \leq 1 \\
1+(\rho-1) \frac{1+|a|}{1-|a|} & \text { if } \rho \geq 1,\end{cases} \\
& \rho_{f}(a, r)= \begin{cases}1+(\rho(a)-1) \frac{r\left(1-|f(a)|^{2}\right)}{r\left(1+|f(a)|^{2}\right)+2|f(a)|} & \text { if } \rho \leq 1 \\
1+(\rho(a)-1) \frac{r\left(1+|f(a)|^{2}\right)+2|f(a)|}{r\left(1-|f(a)|^{2}\right)} & \text { if } \rho \geq 1 .\end{cases}
\end{aligned}
$$

and $\varphi_{b}$ for $b \in \mathrm{D}$ is the Möbius transformation given by

$$
\varphi_{b}(z)=\frac{b-z}{1-\bar{b} z} \quad(z \in \mathrm{D}) .
$$

It is obvious that if $T \in C_{\rho}(H)$ with $\rho>0$ then the operator

$$
T_{a}=\varphi_{a}(T)=(a I-T)(I-\bar{a} T)^{-1}
$$

is well-defined in $B(H)$ for any $a \in \mathrm{D}$. In fact, we have $T_{a} \in C_{\rho(a)}(H)$ (by Theorem 1.1), and $w_{\rho(a)}(T)<1$ if $w_{\rho}(T)<1$ (by Theorem 1.2).

\section{Sharpened von Neumann inequality for strict $\rho$-contractions}

Like in the case $\rho=1$, which was studied by K. Fan [12], [13], the corresponding inequality (1.4) for $a \in \mathrm{D}$ can be sharpened by using an analogue of the Schwarz-Pick inequality for $\rho$-contractions. 
THEOREM 2.1. Let $f$ be a non-constant analytic self map of $\mathrm{D}$, and let $a \in \mathrm{D}$ and $n$ be the the order of multiplicity of the zero a for the function $f-f(a)$. Then for every operator $T$ on $H$ with $w_{\rho}(T)<1$ for some $\rho>0$ we have

$$
w_{\rho(a)}\left[\varphi_{f(a)}(f(T))\right] \leq w_{\rho(a)}\left(T_{a}\right)^{n}
$$

and

$$
w_{\rho_{f}\left(a, w_{\rho(a)}\left(T_{a}\right)^{n}\right)}(f(T)) \leq \frac{w_{\rho(a)}\left(T_{a}\right)^{n}+|f(a)|}{1+w_{\rho(a)}\left(T_{a}\right)^{n}|f(a)|},
$$

where $\rho(a)$ and $\rho_{f}\left(a, w_{\rho(a)}\left(T_{a}\right)^{n}\right)$ are as in (1.6) and (1.7).

Proof. Let $f$ and $a$ as above, and $g=\varphi_{f(a)} \circ f$. Then, we see that

$$
g(z)=\frac{f(a)-f(z)}{1-\overline{f(a)} f(z)}=\frac{a-z}{1-\bar{a} z} h(z) \quad(z \in \mathrm{D})
$$

where $h$ is an analytic function on $\mathrm{D}$ such that $h(\mathrm{D}) \subseteq \mathrm{D}$ (maximum principle). Since $\varphi_{b}^{-1}=\varphi_{b}$, for any operator $T$ with $w_{\rho}(T)<1$ we have

$$
\varphi_{f(a)}(f(T))=g(T)=\varphi_{a}(T) h(T)=\varphi_{a}(T) h_{a}\left(\varphi_{a}(T)\right)=G\left(T_{a}\right),
$$

where $h_{a}=h \circ \varphi_{a}$ and $G(z)=z h_{a}(z)$ for $z \in \mathrm{D}$. As $T_{a} \in C_{\rho(a)}(H)$ and $G(0)=0$, we have $G\left(T_{a}\right) \in C_{\rho(a)}(H)$. Since $r_{a}=w_{\rho(a)}\left(T_{a}\right)<1$ (by (1.4)), we can apply (1.5) to obtain

$$
w_{\rho(a)}\left(G\left(T_{a}\right)\right) \leq M_{G}\left(r_{a}\right) .
$$

But we have

$$
\begin{aligned}
M_{G}\left(r_{a}\right) & =\sup _{|z|=r_{a}}|G(z)|=\sup _{|z|=r_{a}}\left|z h\left(\varphi_{a}(z)\right)\right| \\
& =r_{a} \sup _{|z|=r_{a}}\left|\frac{f(a)-f\left(\varphi_{a}(z)\right)}{1-\overline{f(a)} f\left(\varphi_{a}(z)\right)} \frac{1-\bar{a} \varphi_{a}(z)}{a-\varphi_{a}(z)}\right| \\
& =\sup _{|z|=r_{a}}\left|\varphi_{f(a)}\left(f\left(\varphi_{a}(z)\right)\right)\right| \leq \sup _{|z|=r_{a}}\left|\varphi_{a}\left(\varphi_{a}(z)\right)\right|^{n} \leq r_{a}^{n},
\end{aligned}
$$

the inequality being based on Schwarz-Pick's lemma for derivatives (Lemma 2.1, [1], for instance). Thus, we obtain

$$
w_{\rho(a)}\left[\varphi_{f(a)}(f(T))\right] \leq r_{a}^{n}=w_{\rho(a)}\left(T_{a}\right)^{n},
$$

which is just inequality (2.1). 
Now let us write $S=\varphi_{f(a)}(f(T))$. Then $f(T)=\varphi_{f(a)}(S)$, and since (2.1) yields

$$
w_{\rho(a)}(S) \leq w_{\rho(a)}\left(T_{a}\right)^{n}:=r<1,
$$

from (1.5) we infer

$$
\begin{aligned}
w_{\rho_{f}\left(a, w_{\rho(a)}\left(T_{a}\right)^{n}\right)}(f(T)) & =w_{\rho_{f}\left(a, w_{\rho(a)}\left(T_{a}\right)^{n}\right)}\left(\varphi_{f(a)}(S)\right) \leq \sup _{|z|=r}\left|\varphi_{f(a)}(z)\right| \\
& =\frac{r+|f(a)|}{1+r|f(a)|}=\frac{w_{\rho(a)}\left(T_{a}\right)^{n}+|f(a)|}{1+w_{\rho(a)}\left(T_{a}\right)^{n}|f(a)|},
\end{aligned}
$$

that is the inequality (2.2). The proof is finished.

The sharpened form of the inequality (1.4) with $\rho_{f}=\rho_{f}(0)$ (defined by (1.3)) is obtained in the following

Corollary 2.2. For $f$ and $T$ as in Theorem 2.1 we have

$$
w_{\rho}\left[\varphi_{f(0)}(f(T))\right] \leq w_{\rho}(T)^{n}
$$

and

$$
w_{\rho_{f}\left(0, w_{\rho}(T)^{n}\right)}(f(T)) \leq \frac{w_{\rho}(T)^{n}+|f(0)|}{1+|f(0)| w_{\rho}(T)^{n}},
$$

where either $n=1$, or $n \geq 2$ such that $f^{\prime}(0)=\cdots=f^{(n-1)}(0)=0$.

Proof. One applies (2.1) and (2.2) in the case $a=0$.

Corollary 2.3. Let $f$, $a$ and $n$ be as in Theorem 2.1. Then, for any operator $T$ on $H$ with $\|T\|<1$ we have

$$
\left\|\varphi_{f(a)}(f(T))\right\| \leq\left\|\varphi_{a}(T)\right\|^{n}
$$

and

$$
\|f(T)\| \leq \frac{\left\|\varphi_{a}(T)\right\|^{n}+|f(a)|}{1+|f(a)|\left\|\varphi_{a}(T)\right\|^{n}} .
$$

In particular, for $n$ as in Corollary 2.2 one has

$$
\|f(T)\| \leq \frac{\|T\|^{n}+|f(0)|}{1+|f(0)|\|T\|^{n}} .
$$

We remark that these inequalities for $n=1$ (and $\rho=1$ ) were obtained by K. Fan [12], but his inequalities are weaker than the above ones if $n \geq 2$. We 
also observe that (2.6) yields

$$
|f(z)| \leq \frac{\left|\varphi_{a}(z)\right|^{n}+|f(a)|}{1+|f(a)|\left|\varphi_{a}(z)\right|^{n}} \quad(z \in \mathrm{D}),
$$

if $f, a$ and $n$ are as in Theorem 2.1.

Now, in the case $\rho=2$ we derive from Corollary 2.2 the following

Corollary 2.4. Let $f$ and $n$ be as in Corollary 2.2. If $T \in B(H)$ with $w(T)<1$ then

$$
w_{\rho_{f}\left(0, w(T)^{n}\right)}(f(T)) \leq \frac{w(T)^{n}+|f(0)|}{1+|f(0)| w(T)^{n}} .
$$

Proof. If $\rho=2$ then $\rho_{f}\left(0, w_{\rho}(T)^{n}\right)=\rho_{f}\left(0, w(T)^{n}\right)$, and when $\rho_{f}\left(0, w_{\rho}(T)^{n}\right)=2$ we have $\rho=2\left(w_{\rho}(T)^{n}+|f(0)|\right)\left[w_{\rho}(T)^{n}\left(1+|f(0)|^{2}\right)+\right.$ $2|f(0)|]^{-1}$, so (2.9) obtained from (2.4).

The version for spectral radius of Theorem 2.1 can be also derived.

Corollary 2.5. Let $T$, $a$, and $n$ be as in Theorem 2.1. Then, for any operator $T$ on $H$ with $r(T)<1$ we have

$$
r\left[\varphi_{f(a)}(f(T))\right] \leq r\left(T_{a}\right)^{n}
$$

and

$$
r(f(T)) \leq \frac{r\left(T_{a}\right)^{n}+|f(a)|}{1+|f(a)| r\left(T_{a}\right)^{n}} .
$$

Proof. Since $r(T)<1$, there is $\rho_{0}>1$ such that $w_{\rho}(T)<1$ for $\rho \geq \rho_{0}$. Then we have $\rho_{f}\left(a, w_{\rho(a)}\left(T_{a}\right)^{n}\right) \geq \rho(a) \geq \rho$, hence $\rho(a) \rightarrow+\infty$ and $\rho_{f}\left(a, w_{\rho(a)}\left(T_{a}\right)^{n}\right) \rightarrow+\infty$ if $\rho \rightarrow+\infty$. Thus (2.10) and (2.11) are inferred from (2.1) and (2.2) by letting $\rho \rightarrow+\infty$.

\section{An interior Schwarz inequality for strict $\rho$-contractions}

Under the hypotheses of Theorem 2.1, the inequality (2.3) in the case $f(0)=0$ becomes

$$
w_{\rho}(f(T)) \leq w_{\rho}(T)^{n}
$$

if $w_{\rho}(T)<1$. But this is the Schwarz inequality for $\rho$-contractions in the generalized version for derivatives. In the sequel we will obtain some sharpened forms of this inequality. 
THEOREM 3.1. Let $f$ be a non-constant analytic function such that $f(\mathrm{D}) \subseteq$ $\mathrm{D}$ and $f(0)=f^{\prime}(0)=\cdots=f^{(n-1)}(0)=0$ for some integer $n \geq 1$. Then, for any operator $T$ on $H$ with $w_{\rho}(T)<1$, for some $\rho>0$, and for any $a \in \mathrm{D} \backslash\{0\}$, we have the inequalities

$$
w_{\rho}(f(T)) \leq w_{\rho}(T)^{n} \frac{\left(|a|+w_{\rho}(T)\right)+\left|a^{-n} f(a)\right|\left(1+|a| w_{\rho}(T)\right)}{\left(1+|a| w_{\rho}(T)\right)+\left|a^{-n} f(a)\right|\left(|a|+w_{\rho}(T)\right)}
$$

and

$$
w_{\rho}(f(T)) \leq w_{\rho}(T)^{n} \frac{w_{\rho}(T)+\frac{1}{n !}\left|f^{(n)}(0)\right|}{1+\frac{1}{n !}\left|f^{(n)}(0)\right| w_{\rho}(T)}
$$

Proof. If $f(z)=\lambda z^{n}$ with $|\lambda|=1$ then each of two inequalities one reduces to the power inequality : $w_{\rho}\left(T^{n}\right) \leq w_{\rho}(T)^{n}$, which can also be seen as a particular case of (2.4). Now suppose that $f$ does not have the form $f(z)=\lambda z^{n}$ with $|\lambda|=1$. Define the function $g$ on $\mathbf{D}$ by setting

$$
g(0)=\frac{1}{n !} f^{(n)}(0) \quad \text { and } \quad g(z)=\frac{f(z)}{z^{n}} \quad \text { for } z \in \mathrm{D} \backslash\{0\} .
$$

Then $g$ is an analytic function on $\mathrm{D}$ and $g(\mathrm{D}) \subseteq \mathrm{D}$ because $f(z) \neq \lambda z^{n}$. Thus, if $T \in B(H)$ with $\|T\|<1$ then applying (2.2) for $g$ and $T$ with $\rho=1$ and $a \neq 0$, we obtain

$$
\begin{aligned}
\|f(T)\|=\left\|T^{n} g(T)\right\| & \leq\left\|T^{n}\right\| \frac{\left\|T_{a}\right\|+|g(a)|}{1+|g(a)|\left\|T_{a}\right\|} \\
& \leq\left\|T^{n}\right\| \frac{\left(\frac{|a|+\|T\|}{1+|a|\|T\|}\right)+\left|a^{-n} f(a)\right|}{1+\left|a^{-n} f(a)\right|\left(\frac{|a|+\|T\|}{1+|a|\|T\|}\right)} \\
& =\left\|T^{n}\right\| \frac{(|a|+\|T\|)+|f(a)|\left(\left|a^{-1}\right|+\|T\|\right)}{(1+|a|\|T\|)+|f(a)|\left(1+\left|a^{-1}\right|\|T\|\right)} .
\end{aligned}
$$

Letting $a \rightarrow 0$, one also finds that

$$
\|f(T)\| \leq\left\|T^{n}\right\| \frac{\|T\|+\left|a_{n}\right|}{1+\left|a_{n}\right|\|T\|}
$$

where $a_{n}=(1 / n !) f^{(n)}(0)$. Consequently, the inequalities (3.2) and (3.3) have been proved in the case $\rho=1$. In addition, the above inequalities show for $T=z I$ with $z \in \mathrm{D}$ that

$$
|f(z)| \leq|z|^{n} \frac{(|a|+|z|)+\left|a^{-n} f(a)\right|(1+|a z|)}{(1+|a z|)+\left|a^{-n} f(a)\right|(|a|+|z|)}
$$


for $a \in \mathrm{D} \backslash\{0\}$, and

$$
|f(z)| \leq|z|^{n} \frac{|z|+\left|a_{n}\right|}{1+|z|\left|a_{n}\right|} .
$$

Now let $T \in B(H)$ with $w_{\rho}(T)<1$ for some $\rho>0, \rho \neq 1$. Since $f(0)=0$, we have $\rho^{\prime}=\rho$, and for $a \in \mathbf{D} \backslash\{0\}$ we infer using (1.5) and (3.4) that

$$
\begin{aligned}
w_{\rho}(f(T)) & \leq \sup _{|z|=w_{\rho}(T)}|f(z)| \\
& \leq w_{\rho}(T)^{n} \frac{\left(|a|+w_{\rho}(T)\right)+\left|a^{-n} f(a)\right|\left(1+|a| w_{\rho}(T)\right)}{\left(1+|a| w_{\rho}(T)\right)+\left|a^{-n} f(a)\right|\left(|a|+w_{\rho}(T)\right)}
\end{aligned}
$$

Also, using (1.5) and (3.5) we obtain

$$
w_{\rho}(f(T)) \leq \sup _{|z|=w_{\rho}(T)}|f(z)| \leq w_{\rho}(T)^{n} \frac{w_{\rho}(T)+\left|a_{n}\right|}{1+\left|a_{n}\right| w_{\rho}(T)} .
$$

We conclude that the inequalities (3.2) and (3.4) hold, and this ends the proof.

We remark that (3.3) can be derived from (3.2) taking $a \rightarrow 0$. Both (3.2) and (3.3) are sharpened forms of (3.1) because the factors of the right-hand side of (3.2) and (3.3) are stricly less than 1 , and they are equal to 1 if $f(z)=\lambda z^{n}$ with $|\lambda|=1$.

Having in mind that the inequality (3.5) for $n=1$ is just the interior Schwarz lemma for analytic functions on the unit disc which appears in K. Fan [12], P. Mercer [17] and R. Osserman [19], we can also consider (3.2) and (3.3) as being sharpened forms of the interior Schwarz inequality for $\rho$-contractions.

Now, in the case $n=1$ we obtain from Theorem 3.1 the following result.

Corollary 3.2. Let $f$ be an analytic function $\mathrm{D}$ with $f(\mathrm{D}) \subseteq \mathrm{D}$ and $f(0)=0$. Then, for any operator strict $\rho$-contraction and for $0 \neq a \in \mathrm{D}$ we have

$$
w_{\rho}(f(T)) \leq w_{\rho}(T) \frac{(1+|f(a)|) w_{\rho}(T)+|a|+\left|a^{-1} f(a)\right|}{\left(|a|+\left|a^{-1} f(a)\right|\right) w_{\rho}(T)+1+|f(a)|}
$$

and

$$
w_{\rho}(f(T)) \leq w_{\rho}(T) \frac{w_{\rho}(T)+\left|f^{\prime}(0)\right|}{1+\left|f^{\prime}(0)\right| w_{\rho}(T)} .
$$

In the case $\rho=1$, (3.7) gives just the corresponding inequality of K. Fan [12] for $a=0$, but for $a \in \mathrm{D} \backslash\{0\}$ the inequality of K. Fan is obtained in the 
proof of Theorem 3.1, namely

$$
\|f(T)\| \leq\left\|T^{n}\right\| \frac{\left\|T_{a}\right\|+\left|a^{-1} f(a)\right|}{1+\left|a^{-1} f(a)\right|\left\|T_{a}\right\|}
$$

if $f$ is as in Corollary 3.2. Clearly, this inequality is stronger than (3.6) in this case. Also, for $\rho=1$ we infer from the proof of Theorem 3.1 the following

Corollary 3.3. Let $f$ and $n \geq 2$ be as in Theorem 3.1. Then, for any operator $T$ on $H$ with $\|T\|<1$ and $a \in \mathrm{D} \backslash\{0\}$ we have

$$
\|f(T)\| \leq\|T\|^{n} \frac{(|a|+\|T\|)+\left|a^{-n} f(a)\right|(1+|a|\|T\|)}{(1+|a|\|T\|)+\left|a^{-n} f(a)\right|(|a|+\|T\|)}
$$

and

$$
\|f(T)\| \leq\|T\|^{n} \frac{\|T\|+\frac{1}{n !}\left|f^{(n)}(0)\right|}{1+\frac{1}{n !}\left|f^{(n)}(0)\right|\|T\|}
$$

Finally, letting $\rho \rightarrow+\infty$ in (3.2) and (3.3), we can obtain sharpened forms of the inequality $r(f(T)) \leq r(T)^{n}$ provided by (2.11) when $a=0$ and $f(0)=0$. So we have the following versions for interior Schwarz lemma relatively to the spectral radius.

CoROLlaRY 3.4. Let $f$ and $n$ be as in Theorem 3.1. Then, for any operator $T$ on $H$ with $r(T)<1$ and $0 \neq a \in \mathrm{D}$ we have

$$
r(f(T)) \leq r(T)^{n} \frac{(|a|+r(T))+\left|a^{-n} f(a)\right|(1+|a| r(T))}{(1+|a| r(T))+\left|a^{-n} f(a)\right|(|a|+r(T))}
$$

and

$$
r(f(T)) \leq r(T)^{n} \frac{r(T)+\frac{1}{n !}\left|f^{(n)}(0)\right|}{1+\frac{1}{n !}\left|f^{(n)}(0)\right| r(T)} .
$$

\section{An improved form of the sharpened von Neumann inequality for strict $\rho$-contractions}

Like in the case of contractions [12], [13], the inequalities (2.1) and (2.2) can be sharpened as follows.

THEOREM 4.1. Let $f$ be a non-constant analytic function on $\mathrm{D}$ such that $f(\mathrm{D}) \subseteq \mathrm{D}$ and let $a \in \mathrm{D}$ and $n \geq 1$ be a integer less than or equal to 
the multiplicity of the zero a for the function $f-f(a)$. Then for any strict $\rho$-contraction $T$ on $H$ for some $\rho>0$ we have

$$
w_{\rho(a)}\left[\varphi_{f(a)}(f(T))\right] \leq \beta w_{\rho(a)}\left(T_{a}\right)^{n}
$$

and

$$
w_{\rho_{f}\left(a, \beta w_{\rho(a)}\left(T_{a}\right)^{n}\right)}(f(T)) \leq \frac{\beta w_{\rho(a)}\left(T_{a}\right)^{n}+|f(a)|}{1+\beta|f(a)| w_{\rho(a)}\left(T_{a}\right)^{n}} .
$$

Here $\beta$ can be taken either as $\beta=\beta_{\rho}(f, T, a, n, b)$ with $b \in \mathrm{D}, b \neq a$, or as $\beta=\beta_{\rho}(f, T, a, n)$, where

$$
\begin{aligned}
& \beta_{\rho}(f, T, a, n, b) \\
& =\frac{\left(\left|\varphi_{a}(b)\right|+w_{\rho(a)}\left(T_{a}\right)\right)+\left|\varphi_{a}(b)^{-n} \varphi_{f(a)}(f(b))\right|\left(1+\left|\varphi_{a}(b)\right| w_{\rho(a)}\left(T_{a}\right)\right)}{\left(1+\left|\varphi_{a}(b)\right| w_{\rho(a)}\left(T_{a}\right)\right)+\left|\varphi_{a}(b)^{-n} \varphi_{f(a)}(f(b))\right|\left(\left|\varphi_{a}(b)\right|+w_{\rho(a)}\left(T_{a}\right)\right)}
\end{aligned}
$$

and

$$
\begin{aligned}
\beta_{\rho}(f, T, a, n) & =\frac{w_{\rho(a)}\left(T_{a}\right)+\frac{1}{n !}\left(1-|a|^{2}\right)\left(1-|f(a)|^{2}\right)^{-1}\left|f^{(n)}(a)\right|}{1+\frac{1}{n !}\left(1-|a|^{2}\right)\left(1-|f(a)|^{2}\right)^{-1}\left|f^{(n)}(a)\right| w_{\rho(a)}\left(T_{a}\right)} \\
& =\lim _{b \rightarrow a} \beta_{\rho}(f, T, a, n, b) .
\end{aligned}
$$

Proof. Let us write $F=\varphi_{f(a)} \circ f \circ \varphi_{a}$. Then $F$ is an analytic function on D such that $F(\mathrm{D}) \subseteq \mathrm{D}$ and $F \neq 0$ because $f$ is a non constant function on D. Also, the multiplicity of the zero $z=0$ for $F$ is equal to the multiplicity of the zero $z=a$ for $f-f(a)$. So, by the assumption on integer $n \geq 1$ we have $F(0)=F^{\prime}(0)=\cdots=F^{(n-1)}(0)=0$, and $F$ is not a constant function on D.

Let $T$ be a strict $\rho$-contraction for some $\rho>0$. Then one has $w_{\rho(a)}\left(T_{a}\right)<1$ and $\rho_{F}(a)=\rho(a)$ because $F(0)=0$. We have also $\varphi_{f(a)}(f(T))=F\left(T_{a}\right)$. Now let $b \in \mathrm{D}, b \neq a$ and $\alpha=\varphi_{a}(b)$. Applying the inequality (3.2) with $F$, $T_{a}, \alpha$ and $\rho(a)$ instead of $f, T, a$ and $\rho$ respectively, we get

$$
\begin{aligned}
w_{\rho(a)} & {\left[\varphi_{f(a)}(f(T))\right]=w_{\rho(a)}\left(F\left(T_{a}\right)\right) } \\
& \leq w_{\rho(a)}\left(T_{a}\right)^{n} \frac{\left(|\alpha|+w_{\rho(a)}\left(T_{a}\right)\right)+\left|\alpha^{-n} F(a)\right|\left(1+|\alpha| w_{\rho(a)}\left(T_{a}\right)\right)}{\left(1+|\alpha| w_{\rho(a)}\left(T_{a}\right)\right)+\left|\alpha^{-n} F(a)\right|\left(|\alpha|+w_{\rho(a)}\left(T_{a}\right)\right)} \\
& =\beta_{\rho}(f, T, a, n, b) w_{\rho(a)}\left(T_{a}\right)^{n}
\end{aligned}
$$

where $\beta_{\rho}(f, T, a, n, b)$ is as in Theorem 4.1. On the other hand, applying the inequality (3.3) with $F, T_{a}$ and $\rho(a)$ instead of $f, T$ and $\rho$ respectively, we 
find

$$
\begin{aligned}
w_{\rho(a)} & {\left[\varphi_{f(a)}(f(T))\right]=w_{\rho(a)}\left(F\left(T_{a}\right)\right) } \\
& \leq w_{\rho(a)}\left(T_{a}\right) \frac{w_{\rho(a)}\left(T_{a}\right)+\frac{1}{n !}\left|F^{(n)}(0)\right|}{1+\frac{1}{n !}\left|F^{(n)}(0)\right| w_{\rho(a)}\left(T_{a}\right)} \\
& =w_{\rho(a)}\left(T_{a}\right) \frac{w_{\rho(a)}\left(T_{a}\right)+\frac{1}{n !}\left(1-|a|^{2}\right)\left(1-|f(a)|^{2}\right)^{-1}\left|f^{(n)}(a)\right|}{1+\frac{1}{n !}\left(1-|a|^{2}\right)\left(1-|f(a)|^{2}\right)^{-1}\left|f^{(n)}(a)\right| w_{\rho(a)}\left(T_{a}\right)} \\
& =\beta_{\rho}(f, T, a, n) w_{\rho(a)}\left(T_{a}\right)^{n} .
\end{aligned}
$$

Here we used the known fact (see [1], p. 18) that

$$
F^{(n)}(0)=(-1)^{n+1} \frac{1-|a|^{2}}{1-|f(a)|^{2}} f^{(n)}(a) .
$$

Thus we proved the inequality (4.1), that is, we have

$$
w_{\rho(a)}\left(F\left(T_{a}\right)\right) \leq \beta w_{\rho(a)}\left(T_{a}\right)^{n}:=r_{a}<1,
$$

where $\beta$ can be chosen either as $\beta=\beta_{\rho}(f, T, a, n, b)$, or as $\beta=\beta_{\rho}(f, T$, $a, n)$. Since $f(T)=\varphi_{f(a)}\left(F\left(T_{a}\right)\right)$ and $f(a)=\varphi_{f(a)}(0)$ (which yields $\rho_{f}(a)=$ $\left.\rho_{\varphi_{f(a)}}(0)\right)$, we obtain by $(1.5)$

$$
\begin{aligned}
w_{\rho_{f}\left(a, \beta w_{\rho(a)}\left(T_{a}\right)^{n}\right)}(f(T)) & =w_{\rho_{f}\left(a, \beta w_{\rho(a)}\left(T_{a}\right)^{n}\right)}\left[\varphi_{f(a)}\left(F\left(T_{a}\right)\right)\right] \leq \sup _{|z|=r_{a}}\left|\varphi_{f(a)}(z)\right| \\
& =\frac{r_{a}+|f(a)|}{1+|f(a)| r_{a}}=\frac{\beta w_{\rho(a)}\left(T_{a}\right)^{n}+|f(a)|}{1+|f(a)| \beta w_{\rho(a)}\left(T_{a}\right)^{n}} .
\end{aligned}
$$

Hence the inequality (4.2) holds with the quoted values for $\beta$.

Now we remark that since one has (see [1], p. 20)

$$
\lim _{z \rightarrow 0} \frac{F(z)}{z^{n}}=\frac{F^{(n)}(0)}{n !}
$$

and taking into account the above expressions of $F^{(n)}(0), \beta_{\rho}(f, T, a, n)$ and $\beta_{\rho}(f, T, a, n, b)$ with $a \neq b \in \mathrm{D}$, it follows that

$$
\lim _{b \rightarrow a} \beta_{\rho}(f, T, a, n, b)=\frac{w_{\rho(a)}\left(T_{a}\right)+\frac{1-|a|^{2}}{1-|f(a)|^{2}} \frac{\left|f^{(n)}(a)\right|}{n !}}{1+\frac{1-|a|^{2}}{1-|f(a)|^{2}} \frac{\left|f^{(n)}(a)\right|}{n !} w_{\rho(a)}\left(T_{a}\right)}=\beta_{\rho}(f, T, a, n) .
$$

This concludes the proof. 
Clearly $\beta<1$ in (4.1) and (4.2), hence these inequalities are sharper than the corresponding ones from Theorem 2.1.

In the case $\rho=1$, which implies $\rho(a)=1$, we infer from Theorem 4.1 the following result which completes Theorem 4 in [12].

COROLlary 4.2. Let $f$, $a$ and $n$ be as in Theorem 4.1. Then for any strict contraction $T$ acting on $H$, we have

$$
\left\|\varphi_{f(a)}(f(T))\right\| \leq \beta_{n}\left\|\varphi_{a}(T)\right\|^{n}
$$

and

$$
\|f(T)\| \leq \frac{\beta_{n}\left\|\varphi_{a}(T)\right\|^{n}+|f(a)|}{1+\beta_{n}|f(a)|\left\|\varphi_{a}(T)\right\|^{n}},
$$

where $\beta_{n}=\beta_{1}(f, T, a, n)$.

Note that for $n=1$, the inequalities (4.3) and (4.4) are respectively the inequalities (20) and (22) from [12], where $\beta_{1}=\beta_{1}(f, T, a, 1)$.

Analogous versions of (4.1) and (4.2) for spectral radius can be derived as follows

COROLlaRy 4.3. Let $f$, $a$ and $n$ be as in Theorem 4.1. Then for any operator $T$ on $H$ with $r(T)<1$ we have

$$
r\left[\varphi_{f(a)}(f(T))\right] \leq \beta_{\infty} r\left(T_{a}\right)^{n}
$$

and

$$
r(f(T)) \leq \frac{\beta_{\infty} r\left(T_{a}\right)^{n}+|f(a)|}{1+\beta_{\infty}|f(a)| r\left(T_{a}\right)^{n}}
$$

where $\beta_{\infty}$ can be taken either as $\beta_{\infty}=\lim _{\rho \rightarrow+\infty} \beta_{\rho}(f, T, a, n, b)$ with $a \neq$ $b \in \mathrm{D}$, or as $\beta_{\infty}=\lim _{\rho \rightarrow+\infty} \beta_{\rho}(f, T, a, n)$.

When $r(T)<1$ and $f(T)=0$, we can derive from (4.5) a lower bound for $r\left(T_{a}\right)^{n}$ in terms of $f(a)$ and $f^{(n)}(a)$ for $a \in \mathrm{D}$. This gives also a lower bound for $\left\|T_{a}\right\|^{n}$, and this result in the case $n=1$ one reduces to Corollary 3 [12].

COROLlary 4.4. Let $f$, $a$ and $n$ be as in Theorem 4.1. Let $T$ be an operator on $H$ such that $f(T)=0$ and $r(T)<1$. Then we have

$$
r\left(T_{a}\right)^{n} \geq \gamma(f, a, n) \geq|f(a)|,
$$

where

$$
\gamma(f, a, n)=\left[|f(a)|+\left(\frac{\left(1-|a|^{2}\right)\left|f^{(n)}(a)\right|}{2 n !(1+|f(a)|)}\right)^{2}\right]^{\frac{1}{2}}-\frac{\left(1-|a|^{2}\right)\left|f^{(n)}(a)\right|}{2 n !(1+|f(a)|)} .
$$


In particular, if $T \in B(H)$ is a strict $\rho$-contraction $(\rho>0)$ such that $f(T)=$ 0 , then we have

$$
w_{\rho(a)}\left(T_{a}\right)^{n} \geq \gamma(f, a, n) .
$$

In addition, the second inequality in (4.7) becomes an equality only when either $f(a)=0$, or $f$ is an n-automorphism of $\mathrm{D}$.

Proof. Since $f(T)=0$ one has $r\left[\varphi_{f(a)}(f(T))\right]=|f(a)|$, and so from (4.5) we obtain

$$
|f(a)| \leq r\left(T_{a}\right)^{n} \frac{r\left(T_{a}\right)^{n}+\frac{1-|a|^{2}}{1-|f(a)|^{2}} \frac{\left|f^{(n)}(a)\right|}{n !}}{1+\frac{1-|a|^{2}}{1-|f(a)|^{2}} \frac{\left|f^{(n)}(a)\right|}{n !} r\left(T_{a}\right)^{n}} .
$$

This means that

$$
\begin{aligned}
& |f(a)|\left[1+\frac{1-|a|^{2}}{1-|f(a)|^{2}} \frac{\left|f^{(n)}(a)\right|}{n !} r\left(T_{a}\right)^{n}\right] \\
& \quad \leq r\left(T_{a}\right)^{n}\left[r\left(T_{a}\right)^{n}+\frac{1-|a|^{2}}{1-|f(a)|^{2}} \frac{\left|f^{(n)}(a)\right|}{n !}\right]
\end{aligned}
$$

or equivalently

$$
|f(a)|+\left(\frac{1-|a|^{2}}{2(1+|f(a)|)} \frac{\left|f^{(n)}(a)\right|}{n !}\right)^{2} \leq\left[r\left(T_{a}\right)^{n}+\frac{1-|a|^{2}}{2(1+|f(a)|)} \frac{\left|f^{(n)}(a)\right|}{n !}\right]^{2},
$$

whence the first inequality in (4.7) follows immediately. The second inequality in (4.7) is equivalent to

$$
|f(a)|\left(1-\frac{1-|a|^{2}}{1-|f(a)|^{2}} \frac{\left|f^{(n)}(a)\right|}{n !}\right) \geq 0 .
$$

Here, equality occurs only if either $f(a)=0$ or

$$
|G(0)|=\frac{\left(1-|a|^{2}\right)\left|f^{(n)}(a)\right|}{n !\left(1-|f(a)|^{2}\right)}=1
$$

where $G$ is the analytic function from $\mathrm{D}$ into $\mathrm{D}$ such that $G(z)=F(z) / z^{n}$ for $0 \neq z \in \mathrm{D}$. In this last case, the maximum modulus principle tells us that $G(z)=\lambda$ for some constant $\lambda$ with $|\lambda|=1$, hence $F(z)=\lambda z^{n}$, and consequently $f=\varphi_{f(a)} \circ F \circ \varphi_{a}$, that is $f$ is an $n$-automorphism of $\mathrm{D}$.

Now if $T \in B(H)$ is a strict $\rho$-contraction for some $\rho>0$ then we have $r(T) \leq w_{\rho}(T)<1$ and also $r\left(T_{a}\right) \leq w_{\rho(a)}\left(T_{a}\right)<1$. Thus (4.8) follows from (4.7) and the proof is finished. 
Next we will show that the inequality (3.1) can also be sharpened in some conditions, independent of (3.3).

THEOREM 4.5. Let $f \neq 0$ be an analytic function on $\mathrm{D}$ such that $f(\mathrm{D}) \subseteq \mathrm{D}$ and $f(0)=f^{\prime}(0)=\cdots=f^{(n-1)}(0)=0$ for some integer $n \geq 1$. Suppose that $f$ does not have the form $f(z)=\lambda z^{n}$ with $\lambda$ a unimodular constant. Then for any strict $\rho$-contraction $T$ on $H$ for some $\rho>0$ we have

$$
w_{\rho}(f(T)) \leq w_{\rho}(T)^{n} \frac{\gamma w_{\rho}(T)+\frac{1}{n !}\left|f^{(n)}(0)\right|}{1+\frac{\gamma}{n !}\left|f^{(n)}(0)\right| w_{\rho}(T)}
$$

where $\gamma=\gamma_{\rho}(f, T, n)$ is given by

$$
\gamma=\frac{w_{\rho}(T)+\frac{n !}{n+1}\left[(n !)^{2}-\left|f^{(n)}(0)\right|^{2}\right]^{-1}\left|f^{(n+1)}(0)\right|}{1+\frac{n !}{n+1}\left[(n !)^{2}-\left|f^{(n)}(0)\right|^{2}\right]^{-1}\left|f^{(n+1)}(0)\right| w_{\rho}(T)} .
$$

Moreover, we have $\gamma<1$ unless either $w_{\rho}(T)=0$ or $f(z) / z^{n}$ is an automorphism of $\mathrm{D}$.

Proof. Define the function $g$ on $D$ by setting

$$
g(0)=\frac{1}{n !} f^{(n)}(0) \quad \text { and } \quad g(z)=\frac{f(z)}{z^{n}} \quad \text { if } \quad z \in \mathrm{D} \backslash\{0\} .
$$

Then $g$ is a non-constant analytic function on D such that $g(\mathrm{D}) \subset \mathrm{D}$. So, applying the inequality (4.2) to the function $g$ when $T=z I$ with $z \in \mathrm{D}$, $a=0, n=1$ and $\rho=1$ one obtains

$$
|g(z)| \leq \frac{\beta_{g}(|z|)|z|+|g(0)|}{1+|g(0)| \beta_{g}(|z|)|z|}=\frac{\beta_{g}(|z|)|z|+\frac{1}{n !}\left|f^{(n)}(0)\right|}{1+\frac{1}{n !}\left|f^{(n)}(0)\right| \beta_{g}(|z|)|z|},
$$

where $\beta_{g}(|z|)=\beta_{1}(g, z I, 0,1)$ is given by Theorem 4.1 , that is

$$
\beta_{g}(|z|)=\frac{|z|+\left(1-|g(0)|^{2}\right)^{-1}\left|g^{\prime}(0)\right|}{1+\left(1-|g(0)|^{2}\right)^{-1}\left|g^{\prime}(0)\right||z|}=\frac{|z|+\frac{n !}{n+1} \frac{\left|f^{(n+1)}(0)\right|}{(n !)^{2}-\left|f^{(n)}(0)\right|^{2}}}{1+\frac{n !}{n+1} \frac{\left|f^{(n+1)}(0)\right|}{(n !)^{2}-\left|f^{(n)}(0)\right|^{2}}|z|} .
$$

Since we have

$$
\frac{n !\left|f^{(n+1)}(0)\right|}{(n+1)\left[(n !)^{2}-\left|f^{(n)}(0)\right|^{2}\right]}=\frac{\left|g^{\prime}(0)\right|}{1-|g(0)|^{2}}=|G(0)|
$$

where $G$ is the analytic function on D defined by

$$
G(z)=\frac{\varphi_{g(0)}(g(z))}{z}, \quad z \in \mathrm{D} \backslash\{0\},
$$


by the maximum modulus principle we infer that $|G(0)|<1$ unless $G$ is a constant function, or equivalently $g$ is an automorphism of $\mathrm{D}$. Thus, if $f$ does not have the form $f(z)=z^{n} g(z)$ where $g$ is an automorphism of $\mathrm{D}$, then we have $|G(0)|<1$ which yields $\beta_{g}(|z|)<1$ for any $z \in \mathrm{D}$.

Now let $T \in B(H)$ be a strict $\rho$-contraction for some $\rho>0$. Since $f(0)=0$ one has $\rho^{\prime}=\rho_{f}=\rho$ so that $f(T) \in C_{\rho}(H)$, and using the above estimation of $|g(z)|$ and the inequality (1.5) one obtains

$$
\begin{aligned}
w_{\rho}(f(T)) & \leq \sup _{|z|=w_{\rho}(T)}|f(z)|=\sup _{|z|=w_{\rho}(T)}\left|z^{n} g(z)\right|=w_{\rho}(T)^{n} \sup _{|z|=w_{\rho}(T)}|g(z)| \\
& \leq w_{\rho}(T)^{n} \frac{\beta_{g}\left(w_{\rho}(T)\right) w_{\rho}(T)+\frac{1}{n !}\left|f^{(n)}(0)\right|}{1+\frac{1}{n !}\left|f^{(n)}(0)\right| \beta_{g}\left(w_{\rho}(T)\right) w_{\rho}(T)} \\
& =w_{\rho}(T)^{n} \frac{\gamma w_{\rho}(T)+\frac{1}{n !}\left|f^{(n)}(0)\right|}{1+\frac{1}{n !}\left|f^{(n)}(0)\right| \gamma w_{\rho}(T)},
\end{aligned}
$$

where $\gamma=\beta_{g}\left(w_{\rho}(T)\right)$, that is

$$
\gamma=\frac{w_{\rho}(T)+\frac{n !}{n+1} \frac{\left|f^{(n+1)}(0)\right|}{(n !)^{2}-\left|f^{(n)}(0)\right|^{2}}}{1+\frac{n !}{n+1} \frac{\left|f^{(n+1)}(0)\right|}{(n !)^{2}-\left|f^{(n)}(0)\right|^{2}} w_{\rho}(T)} .
$$

In addition, taking into account the above remark, we conclude that $\gamma<1$ unless either $f$ has the form $f(z)=z^{n} g(z)$ with $g$ an automorphism of $\mathrm{D}$, or $w_{\rho}(T)=0$. This ends the proof.

COROLlary 4.6. Let $f$ and $n$ be as in Theorem 4.5. Then, for any operator $T$ on $H$ with $\|T\|<1$ we have

$$
\|f(T)\| \leq\left\|T^{n}\right\| \frac{\gamma_{1}\|T\|+\frac{1}{n !}\left|f^{(n)}(0)\right|}{1+\frac{1}{n !}\left|f^{(n)}(0)\right| \gamma_{1}\|T\|},
$$

where $\gamma_{1}:=\gamma_{1}(f, T, n)$ is given by (4.10). In addition, $\gamma_{1}<1$ unless either $T=0$, or $f(z) / z^{n}$ is an automorphism of $\mathrm{D}$.

We remark that in the case $n=1,(4.11)$ is just the inequality (36) from [12].

Finally, we can also obtain a version for spectral radius of (4.11) as follows.

COROLlaRY 4.7. Let $f$ and $n$ be as in Theorem 4.5. Then, for any operator $T$ on $H$ with $r(T)<1$ we have

$$
r(f(T)) \leq r(T)^{n} \frac{\gamma_{\infty} r(T)+\frac{1}{n !}\left|f^{(n)}(0)\right|}{1+\frac{1}{n !}\left|f^{(n)}(0)\right| \gamma_{\infty} r(T)}
$$


where

(4.13)

$\gamma_{\infty}:=\lim _{\rho \rightarrow+\infty} \gamma_{\rho}(f, T, n)=\frac{r(T)+\frac{n !}{n+1}\left[(n !)^{2}-\left|f^{(n)}(0)\right|^{2}\right]^{-1}\left|f^{(n+1)}(0)\right|}{1+\frac{n !}{n+1}\left[(n !)^{2}-\left|f^{(n)}(0)\right|^{2}\right]^{-1}\left|f^{(n+1)}(0)\right| r(T)}$.

In addition, $\gamma_{\infty}<1$ unless either $r(T)=0$, or $f(z) / z^{n}$ is an automorphism of D.

Proof. Since $r(T)<1$ we have $w_{\rho}(T)<1$ for sufficiently large $\rho>1$. So, (4.12) and (4.13) follow from (4.9) and (4.10) respectively by letting $\rho \rightarrow$ $+\infty$.

ACKNOWLEDGEMENTS. The research of the second author was supported by the Contract CEX 05-D11-23/2005.

\section{REFERENCES}

1. Bernal Gonzales, L., and Calderon-Moreno, M. C., Two hyperbolic Schwarz lemmas, Bull. Austral. Math. Soc. 66 (2002), 17-24.

2. Berger, C. A., A strange dilation theorem, Notices Amer. Math. Soc. 12 (1965), 590.

3. Carrot, L., Rayon $\rho$-numérique, Thèse, Université Claude Bernard Lyon 1, 2003, 1-109.

4. Cassier, G., Mapping formula for functional calculus, Julia's lemma for operators and applications, Prépublication de l'Institut Camille Jordan, Université Claude Bernard Lyon 1, 2005, 1-22.

5. Cassier, G., and Fack, T., Contractions in von Neumann Algebras, J. Funct. Anal. 135 (1996), 297-338.

6. Cassier, G., and Suciu, N., Mapping theorems and Harnack ordering for $\rho$-contractions, Indiana Univ. Math. J. 55 No 2 (2006), 483-523.

7. Cassier, G., and Suciu, N., Inéalités de von Neumann pondérées, Proc. of the $9^{\text {th }}$ National Conference of the Romanian Mathematical Society, Lugoj, May 2005, Editura Universitatii de Vest Timisoara, 2005, 81-93.

8. Cassier, G., and Suciu, N., Analytic functions of a uniformly stable $\rho$-contraction, Operator Theory 20, Theta Bucharest 2006, 55-73.

9. Dristschel, M. A., Mc Culllough, S., and Woederman, H. J., Model theory for $\rho$-contractions, $\rho \leq 2$, J. Operator Theory 41 (1999), 321-350.

10. Dunford, N., and Schwarz, J. T., Linear Operators, Part I: General Theory, Interscience, New York, 1958.

11. Fan, K., Analytic functions of a proper contraction, Math. Z. 160 (1978), 275-290.

12. Fan, K., Sharpened forms of an inequality of von Neumann, Math. Z. 194 (1987), 7-13.

13. Fan, K., Applications and sharpened forms of an inequality of von Neumann, Current Trends in Matrix Theory, Editors F. Uhlig and R. Grone, Elsevier Science Publishing Co., Inc., 1987, 113-121.

14. Gaspar, D., Spectral $\rho$-dilations for the representations of functions algebras, Analele Univ. Timisoara, Ser. St. Mat. 3 (1970), 153-157.

15. Holbrook, J. A. R., On the power-bounded operators of Sz.-Nagy and Foias, Acta Sci. Math (Szeged) 29 (1968), 299-310.

16. Kalyuzhnyy-Verbovetzkiy, D. S., Multivariable $\rho$-contractions, Oper. Theory Adv. Appl. 160, Birkhäuser Verlag Bassel (2005), 273-298. 
17. Mercer, P. R., Sharpened versions of the Schwarz Lemma, J. Math. Anal. Appl. 205 (1997), 508-511.

18. Neumann, J. von, Eine Spektralentheorie für allgemeine Operatoren eines unitären Raumes, Math. Nachr. 4 (1950/51), 258-281.

19. Osserman, R., A sharp Schwarz inequality of the boundary, Proc. Amer. Math. Soc. 128, 12 (2000), 3513-3517.

20. Sz.-Nagy, B., Sur les contractions de l'espace de Hilbert, Acta. Sci. Math. (1954), 87-92.

21. Sz.-Nagy, B., and Foias, C., On certain class of power bounded operators in Hilbert space, Acta Sci. Math. (Szeged) 27 (1996), 17-25.

22. Sz.-Nagy, B., and Foias, C., Analyse harmonique des opérateurs sur l'espace de Hilbert, Masson, Akadémiai Kiado Budapest, 1967.

23. Williams, J. A. R., Schwarz norms for operators, Pacific J. Math. 24 (1968), 181-188.

UNIVERSITÉ DE LYON, LYON, F-69003, FRANCE; UNIVERSITÉ LYON 1, INSTITUT CAMILLE JORDAN, VILLEURBANNE CEDEX, F-69622, FRANCE; CNRS, UMR 5208 .

E-mail: cassier@math.univ-lyon1.fr
DEPARTMENT OF MATHEMATICS WEST UNIVERSITY OF TIMISOARA BV V. PÂRVAN 4

TIMISOARA 300223

ROMANIA

E-mail: suciu@math.uvt.ro 